\title{
On the Choice of Base Geometry, Type and Design of the Passive Radio Reflector of the Passive Radar Complex
}

\author{
Vitaly V. Loy ${ }^{\mathrm{a} *}$, Evgeny N. Garin ${ }^{\mathrm{a}}$, \\ Aleksey N. Fomin a and Nikita V. Kopilov ${ }^{b}$ \\ ${ }^{a}$ Siberian Federal University \\ Krasnoyarsk, Russian Federation \\ ${ }^{b}$ Yaroslavl Higher Military School of Air Defense \\ Yaroslavl, Russian Federation
}

Received 24.07.2020, received in revised form 24.09.2020, accepted 01.10.2020

Abstract. The article presents the methods that allow to solve the problem of choosing the type, design and installation location of a control terrain feature (CTF) - a passive radio reflector for a passive location complex that implements the angle measuring-range-difference method. The CTF module must reflect the signal at angles from $0^{\circ}$ to $180^{\circ}$ (upper hemisphere). The rationale for choosing the $\mathrm{CTF}$ module and base geometry will allow receiving signals from jammers from any direction relative to the main receiving point.

Keywords: control terrain feature, ellipse, errors, passive radio reflector, active-passive radar system, monostatic RCS, bistatic RCS, corner reflector, bistatic scattering indicatrix, bistatic angle.

Citation: Loy V.V., Garin E.N., Fomin A.N., Kopilov N.V. On the choice of base geometry, type and design of the passive radio reflector of the passive radar complex, J. Sib. Fed. Univ. Eng. \& Technol., 2020, 13(7), 907-918. DOI: 10.17516/1999-494X0277

(C) Siberian Federal University. All rights reserved

This work is licensed under a Creative Commons Attribution-Non Commercial 4.0 International License (CC BY-NC 4.0).

* Corresponding author E-mail address: 1v2009.1974@mail.ru 


\title{
О выборе геометрии базы, типа и конструкции
}

\author{
пассивного радиоотражателя \\ комплекса пассивной радиолокации
}

\author{
В.В. Лойа ${ }^{a}$ Е.Н. Гарин ${ }^{a}$, А.Н. Фомин ${ }^{a}$, Н.В. Копылов ${ }^{0}$ \\ ${ }^{a}$ Сибирский федеральный университет \\ Российская Федераиия, Красноярск \\ бярославское высшее военное училище \\ противовоздушной оборонь \\ Российская Федерачия, Ярославль
}

Аннотация. В статье представлена методика, позволяющая решить задачу выбора типа, конструкции и места установки контрольного местного предмета (КМП) - пассивного радиоотражателя для комплекса пассивной локации, реализующего угломерно-разностнодальномерный метод. Модуль КМП должен обеспечивать отражение сигнала в углах от $0^{\circ}$ до $180^{\circ}$ (верхняя полусфера). Обоснование рационального выбора модуля КМП и геометрии базы позволит принимать сигналы постановщиков активных помех (ПАП) с любого направления относительно основного пункта приема.

Ключевые слова: контрольный местный предмет, эллипс, ошибки, пассивный радиоотражатель, активно-пассивные радиолокационные системы, моностатическая ЭПР, бистатическая ЭПР, уголковый отражатель, бистатическая индикатриса, бистатический угол.

Цитирование: Лой, В.В. О выборе геометрии базы, типа и конструкции пассивного радиоотражателя комплекса пассивной радиолокации / В.В. Лой, Е.Н. Гарин, А.Н. Фомин, Н.В. Копылов // Журн. Сиб. федер. ун-та. Техника и технологии, 2020. 13(7). С. 907-918. DOI: 10.17516/1999-494X-0277

В настоящее время в системе ПВО основное средство воздушной разведки - активные радиолокационные средства. Это предъявляет к ним высокие требования и вызывает дополнительные затраты ресурсов на совершенствование их систем борьбы с активными помехами, что не всегда оправданно при низкой живучести и высокой стоимости данных РЛС.

Главным недостатком существующих однопозиционных радиолокационных систем является низкая радиотехническая скрытность и, как следствие, низкая живучесть. Поэтому перспективными принято считать многопозиционные активно-пассивные радиолокационные системы (МП АП РЛС), использующие либо отраженный зондирующий сигнал (активные однопозиционные РЛС с пассивным ответом), либо собственное радиоизлучение целей (пассивные МП РЛС) [1].

Высокий уровень развития теории пассивной локации позволяет осуществлять обнаружение воздушных объектов по их собственному излучению на дальностях, при которых мощность принятого сигнала может явно не выделяться над уровнем внутренних шумов приемного тракта [2].

При этом реализация методов обнаружения воздушных объектов в комплексах пассивной локации (КПЛ) позволяет обеспечить высокую скрытность и живучесть радиотехнических систем. В работе [3] проведено исследование возможности обнаружения целей, осуществляющих полет в режиме радиомолчания за счет использования сигналов от постановщиков активных

$$
-908-
$$


помех (ПАП) и других источников излучения на основе угломерно-разностно-дальномерного метода. Это связано с тем, что данный метод является энергетически наиболее выгодным из всех существующих методов пассивной локации за счет использования высоконаправленной антенны с большим коэффициентом усиления и при использовании электромагнитной энергии одного ПАП позволяет однозначно оценить координаты любого количества объектов, так как система уравнений имеет единственное решение.

Реализация данного метода предполагает использование цифровой фазированной антенной решетки (ЦФАР), расположенной в основном пункте приема. ЦФАР формирует две ДНА: первая образует целевой канал (ЦК) в направлении на воздушный объект (ВО) или контрольный местный предмет (КМП); вторая образует опорный канал (ОК) в направлении на ПАП. При этом под КМП понимается возвышенность искусственного или естественного происхождения, находящаяся в зоне прямой видимости РЛС и имеющая точно известные координаты, при этом положение энергетических центров «блестящих точек» позволяет однозначно оценить координаты этого КМП.

В соответствии с положениями угломерно-разностно-дальномерного метода пассивной локации на рис. 1 пояснена геометрия определения координат ПАП с одной базой. РЛС, расположенная в основном пункте приема, формирует две достаточно узкие ДНА - на ПАП и КМП. В направлении на ПАП формируется основной канал (ОК) для приема эталонного сигнала для корреляционного обнаружителя.

На КМП формируется целевой канал для приема отраженного сигнала. По сигналу, принятому непосредственно от ПАП, и сигналу, отраженному от КМП, с помощью корреляционного обнаружителя определяется разность расстояний, пройденных сигналами, принятыми напрямую от ПАП и переотраженного от КМП.

В соответствии с расположением объектов ПАП, КМП и РЛС разность расстояний, пройденных сигналами, определяется как

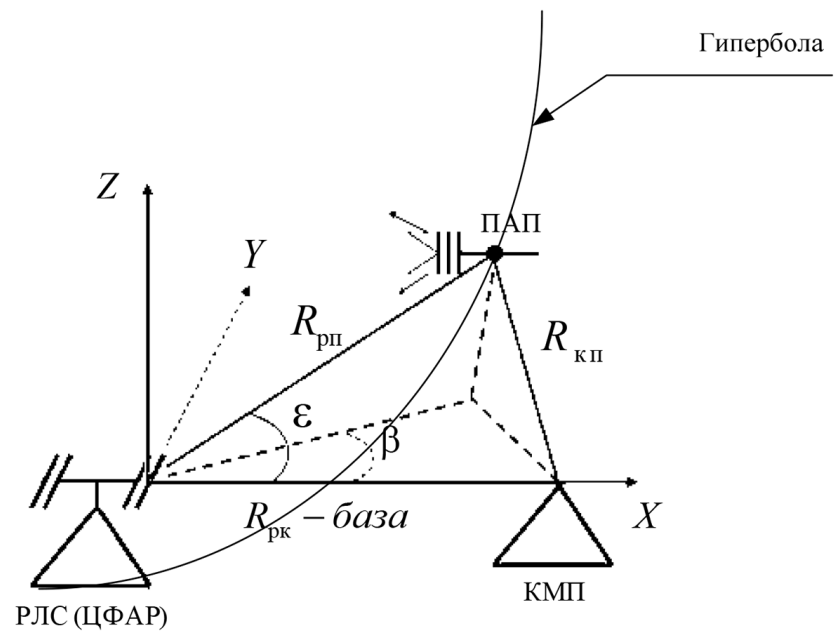

Рис. 1. Геометрия определения координат ПАП угломерно-разностно-дальномерным методом с одной базой

Fig. 1. Geometry for determining PAP coordinates using the single-base angle-difference-rangefinder method

$$
-909-
$$




$$
\Delta r=\left(R_{P K}+R_{\mathrm{K \Pi}}\right)-R_{P \Pi},
$$

где $R_{\text {КП }}$ - расстояние, пройденное сигналом от ПАП до КМП; $R_{\text {РП }}-$ расстояние, пройденное сигналом от ПАП до РЛС.

Таким образом, местоположение ПАП в пространстве соответствует точке пересечения двух плоскостей и поверхности гиперболоида.

$$
R_{\mathrm{P}}=\frac{R_{\mathrm{PK}}^{2}-\Delta r^{2}}{2 \cdot\left(R_{\mathrm{PK}} \cdot \cos (\varepsilon) \cdot \cos (\beta)-\Delta r\right)},
$$

где $\beta$ и $\varepsilon$ характеризуют азимут и угол места ПАП относительно линии базы;

$R_{\text {РК }}-$ база системы (Б), расстояние от точки стояния РЛС до КМП;

$\Delta r$ - разность расстояний, пройденных сигналами от ПАП до РЛС и переотраженного сигнала от КМП в направлении на РЛС.

Таким образом, по переотраженной от КМП электромагнитной энергии ПАП определяется местоположение самого ПАП, которое оценивается по угловому положению ДНА ( $\beta$ и $\varepsilon)$ целевого канала (ЦК) и наклонной дальности до ПАП $R_{\text {РП }}[4]$.

Важной и актуальной задачей при реализации данного метода определения местоположения ПАП является выбор типа, конструкции и места установки КМП (пассивного отражателя). Методика решения подобной задачи была предложена и исследована в работе [5].

В частности, в качестве пассивного радиоотражателя как с энергетической, так и с экономической точки зрения было предложено использовать уголковый радиоотражатель (УРО), дающий хорошее отражение в широком диапазоне углов падения приходящего радиосигнала. ЭПР треугольного УРО зависит от длины его ребра и длины волны и рассчитывается по формуле

$$
\sigma_{\mathrm{yO}}=\frac{4 \pi \cdot a^{4}}{3 \cdot \lambda^{2}}
$$

где $a$ - длина ребра, $\lambda$ - длина волны.

Для увеличения ЭПР несколько УРО объединяют в один конструктивный модуль. Варианты компоновки модуля УРО для КМП будут рассмотрены ниже. Чтобы произвести расчет требуемой величины ЭПР УРО и его конфигурации, необходимо произвести расчет радиолинии ПАП-КМП-РЛС, для чего воспользуемся методикой, изложенной в работе [4].

Задав в качестве первого приближения вероятность правильного обнаружения равной 0,9 и вероятность ложной тревоги $10^{-6}$ при отношении сигнал/шум равным 10 , можно определить максимальную дальность определения местоположения ПАП в соответствии с уравнением радиосвязи для разнесенных приемных и передающих пунктов [6] как

$$
R_{\mathrm{P} \Pi \max }=\sqrt{\frac{P_{\Pi \mathrm{I} \Pi} \cdot G_{\Pi \mathrm{I} \Pi} \cdot \sigma_{K M I} \cdot A_{Э \Phi}}{(4 \cdot \pi)^{2} \cdot P_{\text {umin }} \cdot R_{\mathrm{PK}}^{2}}},
$$

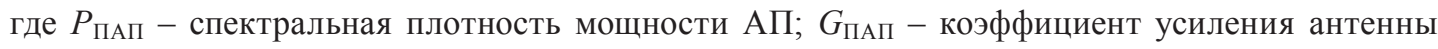

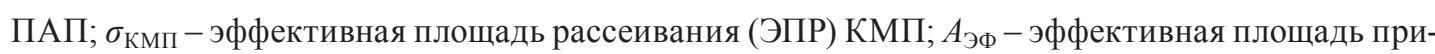
емной антенны РЛС; $P_{Ц \text { min }}-$ минимальная мощность сигнала, достаточная для обнаружения ПАП; $R_{\text {РК }}$ - база системы (Б), расстояние от точки стояния РЛС до КМП.

$$
-910-
$$


Согласно расчетам, приведенным в [3, 4] для максимальной дальности определения местоположения ПАП при длине волны $\lambda=0,23$ м, величина ЭПР КМП должна быть в пределах $50-60 \mathrm{M}^{2}$. При этом КМП должен располагаться на удалении $10-30$ км от РЛС в зоне прямой видимости.

\section{Выбор типа и облика пассивного радиоотражателя}

Модуль УРО должен обеспечивать отражение сигнала в углах от $0^{\circ}$ до $180^{\circ}$ (верхняя полусфера). Компоновка из четырех УРО (рис. 2) позволит отражать сигналы ПАП при всевозможных положениях модуля относительно постановщика помех [5]. Недостатком такой компоновки является провал ЭПР при нахождении ПАП в зените над модулем УРО.

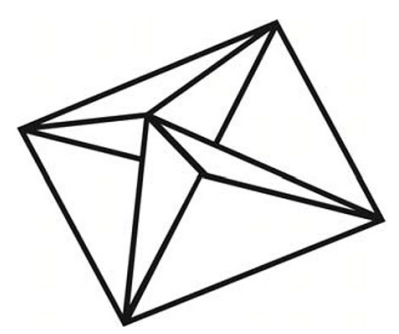

Рис. 2. Трехмерная модель модуля из четырех УРО

Fig. 2. A three-dimensional model of a module of four URO

На рис. 3 представлен вариант компоновки модуля УРО из четырех треугольных УРО и одной двухгранной пластины посередине. Такая конструкция модуля УРО позволит избежать отмеченного выше недостатка.

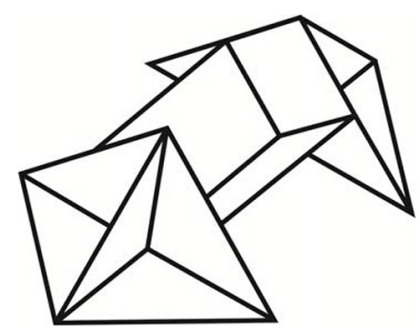

Рис. 3. Трехмерная модель модуля из четырех УРО и одной двухгранной пластины

Fig. 3. Three-DIMENSIONAL model of the module consisting of four URO and one two-sided plate

В зависимости от формы граней различают треугольные (a), прямоугольные (б) и круглые (в) уголковые отражатели (рис. 4). Их максимальные ЭПР соответственно равны:

$$
\sigma_{\Delta}=\frac{4}{3} \pi \frac{a^{4}}{\lambda^{2}}
$$




$$
\begin{aligned}
& \sigma_{\square}=12 \pi \frac{a^{4}}{\lambda^{2}}, \\
& \sigma_{o}=2 \pi \frac{a^{4}}{\lambda^{2}},
\end{aligned}
$$

где а - длина ребра отражателя; $\lambda$ - длина волны.

Уголковые отражатели с малыми размерами дают весьма высокую ЭПР. Так, при $\lambda=3$ см и $a=50 \mathrm{~cm} \sigma_{\square}=2500 \mathrm{M}^{2}$.

Сектор переизлучения уголковых отражателей на уровне половинной мощности составляет примерно 40 - 50 град.

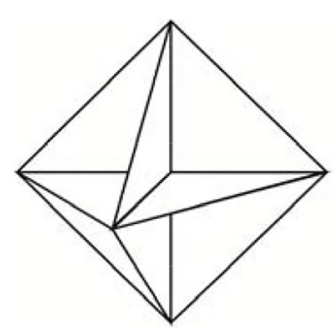

a

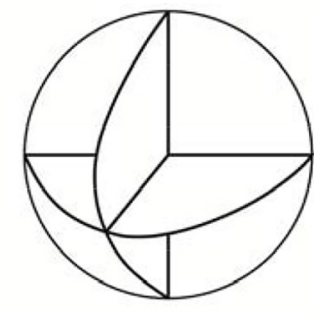

б

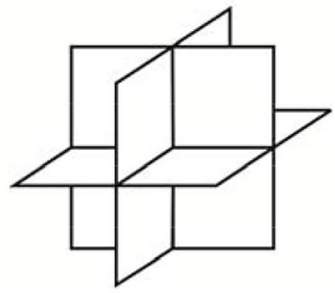

Рис. 4. Конструкции уголковых отражателей

Fig. 4. Design corner reflectors

Важно отметить, что все рассуждения и расчеты, проведенные выше и касающиеся величины ЭПР пассивного радиоотражателя (КМП), выбора его типа и конструкции, справедливы для понятия моностатической ЭПР [1]. ЭПР объекта, в общем случае, зависит как от формы, размеров, материала, из которого он изготовлен, длины волны зондирующего сигнала, так и от направления падающей на него электромагнитной волны. Конструкции уголковых отражателей имеют высокую величину ЭПР, как показано в работе [7] для моностатического режима; рассчитанное значение величины ЭПР обеспечивается при совпадении направлений от рассеивателя (в нашем случае от КМП) и направления в точку приема переотраженного сигнала. Согласно иллюстрации, представленной на рис. 5, для уголкового отражателя падающая и отраженная электромагнитные волны для получения максимальной ЭПР должны лежать в одной плоскости (рис. 5).

В рассматриваемом нами методе определения местоположения ПАП в пространстве источник радиосигнала (ПАП) и точка приема переотраженного сигнала от КМП (РЛС см. рис. 1) разнесены в пространстве, образуя между направлениями $R_{\mathrm{P}}$ и $R_{\mathrm{PK}}$ так называемый бистатический угол, т.е. это угол между направлениями излучения и приема.

Зависимости интенсивности, плотности потока энергии рассеянного поля или ЭПР от углов облучения или приема носят название индикатрис рассеяния. Согласно терминологии, представленной в работе [8], в рассматриваемом методе имеет место пространственная бистатическая индикатриса рассеяния. Следует отметить, что пространственные индикатрисы рассеяния или полный набор индикатрис на стандартных поляризациях в подавляющем 


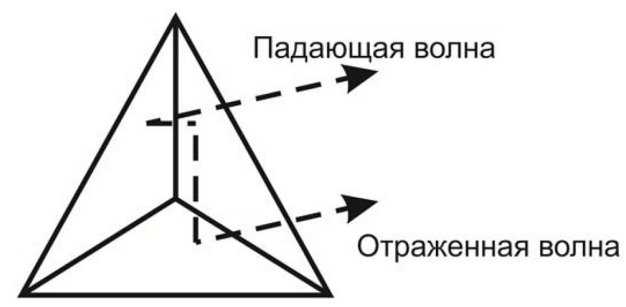

Рис. 5. Направления падающей и отраженной волн от уголкового отражателя

Fig. 5. Directions of incident and reflected waves from the angle reflector

большинстве случаев неизвестны даже для тел простой формы, не говоря уже о специальных отражателях. Исключение составляет единственный радиолокационный отражатель (как показали результаты проведенных авторами патентных исследований) - это сфера [8].

Задача о рассеянии и дифракции плоской электромагнитной волны на сфере исследована наиболее полно по сравнению со всеми другими телами простой и сложной формы. Число опубликованных к настоящему времени работ, касающихся дифракции на сфере, очень велико. Их начало было положено классическими работами Шварцшильда, Ми и Дебая. Интерес к задаче дифракции на сфере вполне понятен. Многие явления, начиная от рассеяния света и кончая распространением радиоволн, в той или иной степени связаны с этой задачей.

Особенно велико ее значение для радиолокации, поскольку сфера представляет собой одно из тел простой формы, для которого задача решается совершенно строго. По этой причине металлические сферы широко используют в качестве эталонов ЭПР.

Кроме того, сфера обладает одним уникальным свойством: это единственное тело, рассеивающее энергию во все стороны равномерно [8].

Иными словами, сфера является всенаправленным отражателем как в моностатическом, так и в бистатическом режиме. Моностатическая всенаправленность сферы очевидна и не требует пояснений.

Что же касается бистатической всенаправленности, то иногда, по словам автора работы [8], этот вопрос вызывает недоумение и нуждается в доказательстве. Подробное доказательство данного положения рассмотрено в [8] в приближении к задачам геометрической оптики [9].

В большинстве случаев, когда радиус сферы много больше длины волны падающего электромагнитного поля $(a / \lambda)$, бистатическая ЭПР сферы при полном поляризационном приеме соответствует выражению (8)

$$
\sigma_{m}=\pi a^{2}
$$

где $a$ - радиус сферы.

График энергетической функции рассеяния, рассчитанный в работе [10], приведен на рис. 6. В резонансной области ЭПР сферы осциллирует, приближаясь к асимптотическому значению $\sigma_{m}=\pi a^{2}$ при увеличении $2 \mathrm{a} / \lambda$.

Наибольшее относительное значение ЭПР, равное $3,65 \sigma_{m}$, наблюдается при $2 a \approx 0,326 \lambda$, что примерно соответствует условию $k a=1$. На рис. 6 указаны выраженные в процентах отклоне- 


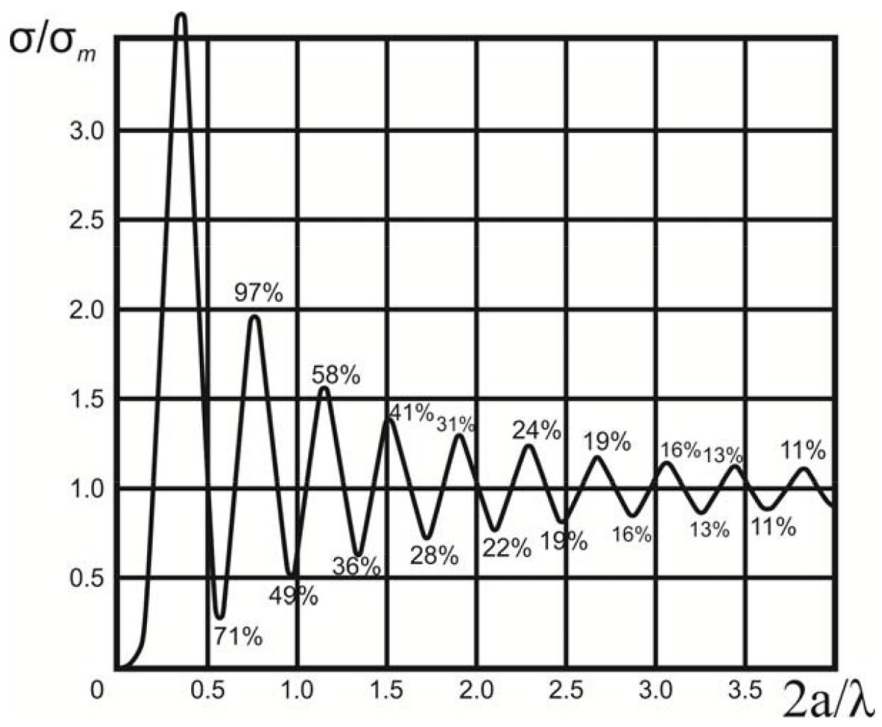

Рис. 6. Энергетическая функция рассеяния сферы

Fig. 6. Energy scattering function of a sphere

ния последующих максимумов и минимумов от асимптотического значения. Отклонения не превосходят $10 \%$ при $k a \geq 12 k=2 \pi / \lambda$ - волновое число.

Таким образом, оптимальным вариантом выбора пассивного радиолокационного радиоотражателя для нашего случая следует считать сферу.

График зависимости, рассчитанный с помощью программы для ЭВМ [11], приведен на рис. 7. Для обеспечения максимальной дальности определения местоположения ПАП величина ЭПР КМП, согласно выражению (4) и проведенному математическому моделированию с помо-

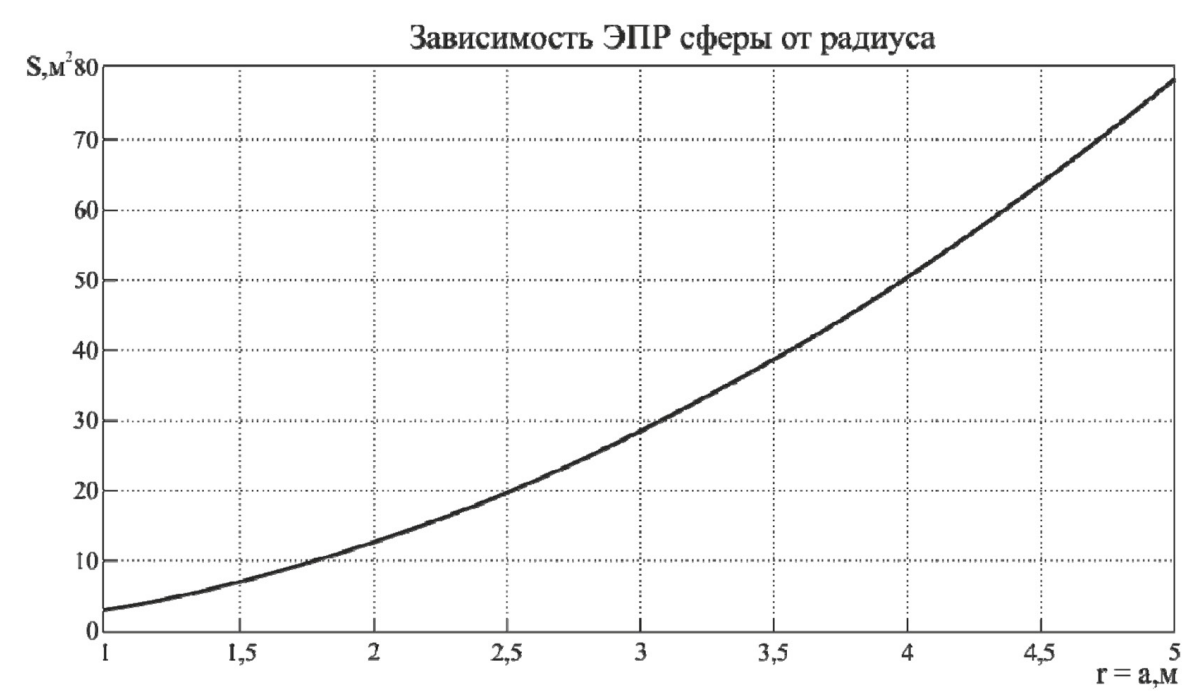

Рис. 7. График зависимости ЭПР КМП от радиуса сферы

Fig. 7. A graph of EPR of the KMP from the radius of the sphere 
щью программы для ЭВМ [11], находится в пределах 50-75 м². При этом радиус сферы лежит в пределах 4-5 м.

\section{Выбор геометрии базы, количества КМП и мест их размещения на местности относительно основного пункта приема}

Представленная на рис. 1 геометрия расположения на местности основного пункта приема (РЛС ЦФАР) и КМП позволяет решать задачу определения координат ПАП угломерноразностно-дальномерным методом с одной базой в широком секторе углов в горизонтальной плоскости, а в вертикальной - на малых углах места ограничивается дальностью прямой видимости. При этом расположение КМП относительно РЛС также предполагает условие дальности прямой видимости в пределах 10-30 км от РЛС в зависимости от состояния местности. Вопросы, связанные с расчетом многопозиционных систем по точностным и разрешающим характеристикам, достаточно подробно описаны в работе [1]. Исходя из приведенных в [1] практических рекомендаций, считают целесообразным при многопозиционном построении РЛС выбирать два вспомогательных приемных пункта (в нашем случае два КМП), расположенных на взаимно перпендикулярных базах относительно основного (РЛС).

Наличие в рассматриваемой пассивной бистатической системе высокопотенциальной РЛС с цифровой ФАР позволяет однозначно измерять углы (пеленги) на ПАП - азимутальный $\beta$ и угломестный $\varepsilon$. Точность и разрешающая способность по угловым координатам зависит от ширины диаграммы направленности РЛС в соответствующей плоскости (рис. 8а).

При наличии одного КМП максимальная точность определения координат будет в том случае, когда ПАП находится на линии в плоскости $Z O X$, перпендикулярной относительно центра базы [1], - минимальные размеры эллипса ошибок (рис. 8а). Размеры эллипса ошибок в горизонтальной плоскости (малой и большой полуосей) определяются мерой разрешения РЛС по азимуту $\delta \beta$ и мерой разрешения по разности дальностей $\Delta r=c / \Delta f_{c}$, где $\Delta f_{c}$ - ширина спектра помехового сигнала ПАП, $c$ - скорость света [1].

По мере удаления ПАП от линии проекции на центр базы (влево или вправо) на рис. $8 \mathrm{a}$ обозначены цифрами: 1 - по центру базы; 2-4-смещение ПАП вправо, размеры эллипса ошибок увеличиваются и, соответственно, ухудшается точность определения координат ПАП.

И наконец, когда местоположение ПАП окажется на линии ПАП-КМП-РЛС (на рис. 8а точка 4), размеры эллипса ошибок по большей полуоси уходят на бесконечность - (рис. 8б). В этом случае возникает ситуация неопределенности; когда определение местоположения ПАП становится невозможным. По этой причине для устранения возникшей неопределенности предложено использовать два КМП с взаимно-перпендикулярным расположением их базы на местности относительно точки стояния РЛС (рис. 8в).

Алгоритм вычисления разности расстояний $\Delta \mathrm{r}$, входящий в выражение (1), в этом случае предполагает использование переизлученного сигнала ПАП от КМП 1 при нахождении ПАП в верхней полусфере в пределах углов от $0^{\circ}$ до $180^{\circ}$ или же от 2-го КМП при нахождении ПАП в нижней полусфере в пределах углов от $180^{\circ}$ до $0^{\circ}$ (рис. 8в). Информация о нахождении ПАП в той или другой полусфере заключена в значении угла (азимута) по результатам пеленгации.

Таким образом, описанная выше методика позволяет решить задачу выбор типа, конструкции и места установки КМП (пассивного радиоотражателя) для комплекса пассивной 


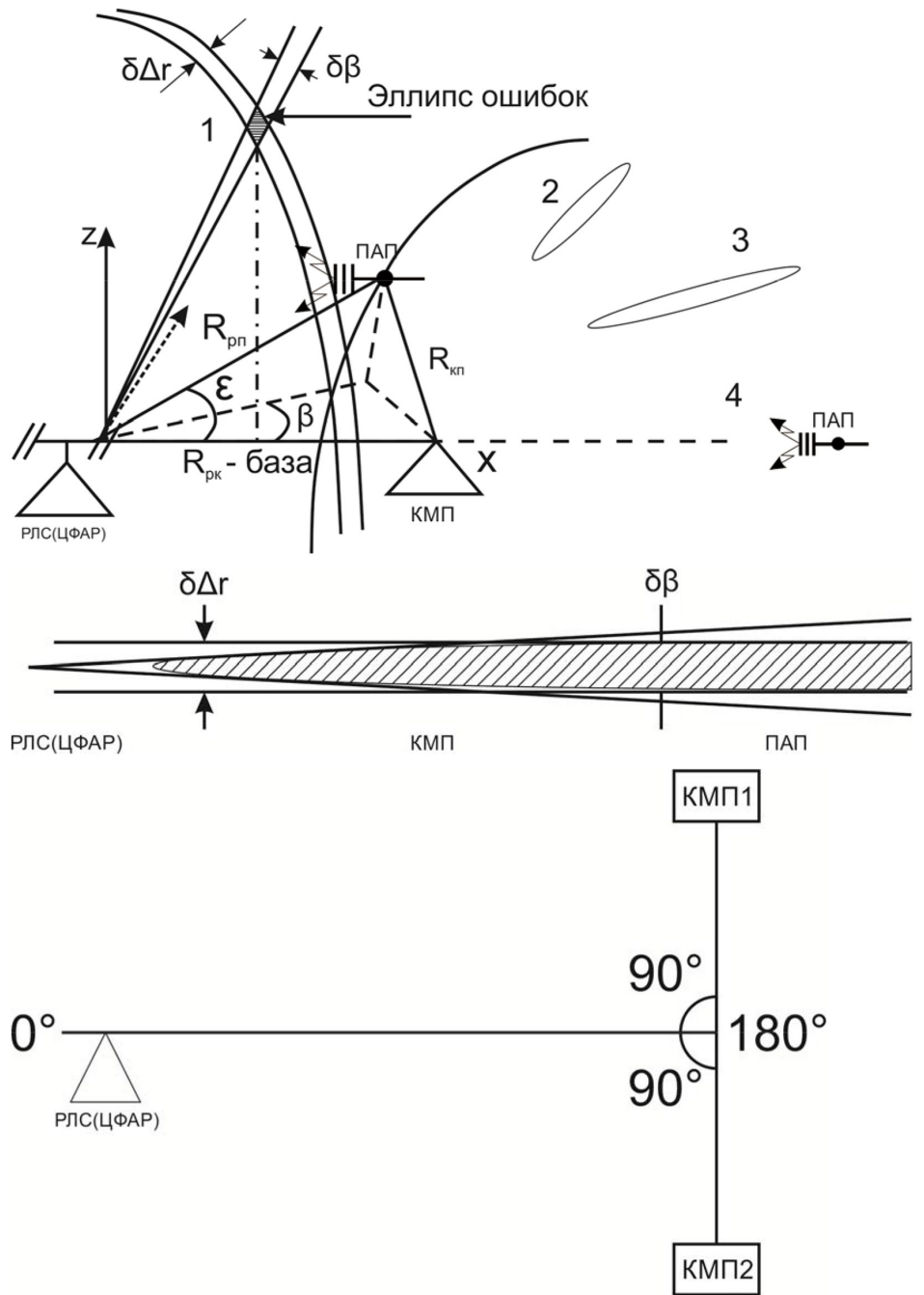

Рис. 8. Пассивная бистатическая РЛС с двумя пассивными радиоотражателями

Fig. 8. Passive bistatic radar with two passive radio reflector

локации, реализующего угломерно-разностно-дальномерный метод. Предлагаемая геометрии базы, количество КМП и места их размещения на местности относительно основного пункта приема позволят, на наш взгляд, устойчиво принимать сигналы постановщиков активных помех с любого направления относительно основного пункта приема и определять местоположение самого ПАП в пространстве.

Для подтверждения положительных выводов предложенной методики и ее работоспособности в дальнейшем необходимо проведение экспериментальных измерений ЭПР пассивного радиоотражателя (КМП) типа «сфера» во всем интервале углов в лабораторных или полигонных условиях, что является предметом последующих научных исследований. 


\section{Список литературы / References}

[1] Охрименко А.Е. Угловое разрешение целей в многопозиционных радиолокационных системах. Научно-производственное республиканское унитарное предприятие «Алеврут». Минск, Беларусь, 2009. [Okhrimenko A.E. Angular resolution of targets in multi-position radar systems. Scientific production Republican unitary enterprise “will Aliroot”. Minsk, Belarus, 2009. (in Russian)]

[2] Ширман Я.Д. Радиоэлектронные системы: основы построения и теория. Справочник. М.: ЗАО Маквис, 1998. [Shirman J.D. Electronic systems: fundamentals of construction and theory. Reference book. M.: CJSC Makvis, 1998 (in Russian)]

[3] Лой В.В., Каримуллин М.Р. Оптимальный алгоритм обнаружения корреляционно-базовым средством пассивной локации сигнала постановщика активных шумовых помех, переотраженного от воздушного объекта. Всероссийская военно-научная конференция (23 марта 2018 года, Тверь). Сборник материалов (№1) Секция 5. Направления развития формы и способов боевого применения радиотехнических формирований с учетом опыта боевого применения РТВ в Сирийской Арабской Республике. Тверь: ВА ВКО, 2018. С. 428-434. Инв. 61982в. [Loy V.V., Karimullin M.R. Optimal algorithm for detecting the correlation-base means of passive location of the signal of the producer of active noise interference, re-reflected from an air object. All-Russian military scientific conference (March 23, 2018, Tver). Collection of materials (No. 1) Section 5. Directions of development of the form and methods of combat use of radio-technical formations, taking into account the experience of combat use of RTV in the Syrian Arab Republic. Tver: VA VKO, 2018. P. 428-434. Inv. 61982v. (in Russian)]

[4] Кобзарев В.А. Патент 2240576 (РФ) от 20.11.04 г, МПК7 G 01 S 13/06. Способ обнаружения и определения местоположения воздушных объектов / Заявка 20022120568/09. Приоритет 29.07.2002 г. [Kobzarev V.A. Patent 2240576 (RF) dated 20.11.04, IPC7 G 01 S 13/06. Method for detecting and determining the location of air objects / Application 20022120568/09. Priority 29.07.2002 (in Russian)]

[5] Грицан О.Б., Бадертдинов А.М., Чеботарев В.Е., Фомин А.Н. Спутниковая радиоизмерительная система с уголковыми радиоотражателями. Сборник материалов Международной Сибирской конференции по контролю и связи (СИБКОН-2013). Раздел 2. 12-13 сентября, г. Красноярск, 2013 г. [Gritsan O.B., Badretdinov A.M., Chebotarev V.E., Fomin A.N. Satellite radio measuring system with angular radio reflectors. Proceedings of the International Siberian conference on control and communication (SIBCON-2013). Section 2. September 12-13, Krasnoyarsk, 2013 (in Russian)]

[6] Бердышев В.П., Куликов В.Н., Кузнецов В.В. и др. Системотехнические основы построения вооружения радиотехнических войск. Часть 2. Воздействие пассивных помех и защита от них. Основы построения обзорных радиотехнических средств. Под общ. ред. В.П. Бердышева. Тверь: ВА ВКО, 2008.248 c. [Berdyshev V.P., Kulikov V.N., Kuznetsov V.V., etc. System-Technical bases of construction of armament of radio-Technical troops. Part 2. Exposure to and protection from passive interference. Fundamentals of building survey radio equipment. Under the General ed. V.P. Berdysheva. Tver: VA VKO, 2008. 248 p. (in Russian)]

[7] Луценко И.В., Попов И.В., Луценко В.И. Бистатические РЛС с подсветкой ионосферными сигналами связных станций коротковолнового диапазона. Радиофизика и электроника, 2007, 12, 1, $199-203$. ISSN 1028-821X. [Lutsenko I.V., Popov I.V., Lutsenko V.I. Bistatic radars with illumination by ionospheric signals of short-wave communication stations. Radiophysics and electronics, 2007, 12, 1, 199-203. ISSN 1028821X. (in Russian)]

[8] Кобак В.О. Радиолокационные отражатели. М.: Сов. радио,1975, 248 с. [Kobak V.O. Radar reflectors. M., Sov. Radio, 1975, 248 p. (in Russian)] 
[9] Потехин А.И. Некоторые задачи дифракции электромагнитных волн. М.: Сов. радио. 1948, 136 с. [Potekhin A.I. Some problems of diffraction of electromagnetic waves. M., Sov. radio, 1948, 136 p. (in Russian)]

[10] Appel-Hansen J., Mersk-Muller O. Back - scattering cross sections of spheres for calibrating purposes in scattering measurements. Laboratory of Electromagnetic Theory. The Technical University of Denmark Lyngby, 1970, R-79, July, 1.

[11] Лой В.В., Бердышев В.П., Фомин А.Н. и др. Программа расчета ЭПР объектов различной формы для различных длин волн. Свидетельство о государственной регистрации программы для ЭВМ № 2019615074. [Loy V.V., Berdyshev V.P., Fomin A.N. et al. Program for calculating the ESR of objects of different shapes for different wavelengths. Certificate of state registration of the computer program no. 2019615074 (in Russian)] 